\title{
A Lower Bound on Neutrino Mass and Its Implication on The Z-burst Scenario
}

\author{
Kwang-Chang La: * \\ Department of Physics, National Taiwan University, Taipei, Taiwan. \\ Pisin Chent \\ Kavli Institute for Particle Astrophysics and Cosmology, \\ Stanford Linear Accelerator Center, Stanford University, Stanford, CA 94309
}

(Dated: November 7, 2018)

\begin{abstract}
We show that the cascade limit on ultra high energy cosmic neutrino (UHEC $\nu$ ) flux imposes a lower bound on the neutrino mass provided that super-GZK events of ultra high energy cosmic rays (UHECRs) are produced from Z-bursts. Based on the data from HiRes and AGASA, the obtained neutrino mass lower bound violates its existing cosmological upper bound. We conclude that the Zburst cannot be the dominant source for the observed super-GZK UHECR events. This is consistent with the recent ANITA-lite data.
\end{abstract}

PACS numbers:

\section{INTRODUCTION}

Big bang cosmology predicts the existence of both cosmic microwave background (CMB) and cosmic neutrino background $(\mathrm{C} \nu \mathrm{B})$. Ultra high energy cosmic protons are expected to interact effectively with the CMB photons, predominantly through the photopion production at $\Delta$-hyperon resonance, and would lose their energies rapidly with the attenuation length around $50 \mathrm{Mpc}$. As such the ultra high energy cosmic ray (UHECR) spectrum is predicted to exhibit a cutoff the so called GZK cutoff [1, 2] — around the threshold energy $\sim 4 \times 10^{19} \mathrm{eV}$. While observations from the HiRes experiment is consistent with the notion of GZK cutoff 3], the AGASA data appears to suggest the opposite [4, 5]. This leads to many speculations as to whether the GZK cutoff really exists, and if not what is the nature of these super-GZK events.

Existing models for super-GZK UHECRs are usually categorized into top-down and bottom-up scenarios. The top-down scenario assumes the existence of supermassive exotic elementary particles based on theories beyond the standard model. The major challenge of this scenario lies in the demand for a fine-tuned decay and/or annihilation rate and the lack of physical evidence for their existence. On the other hand, the bottom-up scenario, which assumes ordinary particles as the UHECRs, faces the challenge of providing an effective mechanism to accelerate particles to ultra high energies. Even if an effective "cosmic accelerator" can be identified, the issue of cosmic transport dictated by the GZK mechanism remains, as there does not seem to exist identifiable sources within our local super cluster $(\sim 50 \mathrm{Mpc})$ for the detected events.

\footnotetext{
*Electronic address: kclai@phys.ntu.edu.tw

${ }^{\dagger}$ Electronic address: chen@slac.stanford.edu
}

To circumvent this difficulty, it was suggested that the Z-burst, the resonant annihilation of the ultra high energy cosmic neutrino (UHEC $\nu$ ) with the $\mathrm{C} \nu \mathrm{B}$ into a $\mathrm{Z}$ boson and its subsequent decay into ultra high energy protons [6, 7, [8], that occurs within our local super cluster can account for UHECRs beyond the GZK-cutoff [9, 10]. With its mean-free-path comparable to the present Hubble radius, the $\mathrm{UHEC} \nu$ serves as a cosmic messenger that can avoid the GZK proton attenuation problem without invoking particle theory beyond the standard model. For the Z-burst to happen, the $\mathrm{UHEC} \nu$ must be at a resonant energy,

$$
E_{\text {res }}=\frac{M_{Z}^{2}}{2 m_{\nu}} \cong 4 \times 10^{21}\left(\frac{1 \mathrm{eV}}{m_{\nu}}\right) \mathrm{eV},
$$

which depends on the neutrino rest mass, $m_{\nu}$. Here $M_{Z}$ denotes the mass of the $\mathrm{Z}$ boson. If the $\mathrm{Z}$-burst mechanism is indeed responsible for the observed UHECR super-GZK spectrum, then there must exist a constraint on the neutrino mass via the above relation.

Based on the Z-burst scenario, two groups have derived bounds on neutrino masses using AGASA data with different strategies. Fodor, Katz and Ringwald 11] deduce the Z-burst spectrum from the AGASA data by parameterizing the transition from the non-burst to the burst component near and above the "ankle" of the UHECR spectrum. Gelmini, Varieschi and Weiler [12] derive their bound by requiring the Z-burst not to overproduce non-observation events beyond the AGASA end-point energy. Our strategy, instead, is to invoke an upper limit on the UHEC $\nu$ flux so as to obtain an upper bound on the required resonant energy $E_{r e s}$, which can in turn be translated into a lower bound on the neutrino mass.

In this paper we derive a lower bound on the neutrino mass based on the assumption that Z-burst mechanism saturates the observed UHECR super-GZK spec- 
trum. Our deduced neutrino mass lower bound, however, turns out to be higher than the existing upper bound deduced from cosmological considerations. We thus conclude that the Z-burst mechanism cannot be responsible for the super-GZK UHECR spectrum. Our conclusion agrees with that from the recent ANITA-lite experiment [13].

\section{UHECR FLUX AND Z-BURST}

Assume that all observed super-GZK UHECR proton events are induced from Z-bursts. The observed superGZK proton flux must be smaller than the total Z-burst proton yield in the universe since there must be events occurred outside our local GZK-sphere ( $50 \mathrm{Mpc})$ which could not reach the Earth. Furthermore, in order for Z-burst events to saturate the observed super-GZK spectrum, it is inevitable that they are oversupplied since there must be some Z-burst protons that are generated at energies below the GZK-cutoff. Therefore,

$$
I_{p,>G Z K}^{o b s} \leq I_{p \mid \nu}^{Z}
$$

where $I_{p,>G Z K}^{o b s}$ is the total observed super-GZK proton flux with energy exceeding the GZK-cutoff and $I_{p \mid \nu}^{Z}$ the total proton flux from Z-bursts, both in units of $\mathrm{cm}^{-2} \mathrm{~s}^{-1} \mathrm{sr}^{-1}$.

Though observations [14] cannot completely rule out the possible contribution to the super-GZK UHECR spectrum by UHE photons, experimental data 14] suggests that protons saturate the super-GZK flux, $I_{p,>G Z K}^{o b s}=I_{>G Z K}^{o b s}$, at $2 \sigma$ confidence level. Then in terms of the total observed UHECR flux, Eq.(2) can be written as

$$
I_{>G Z K}^{o b s} \leq I_{p \mid \nu}^{Z}
$$

The AGASA experiment has accumulated 57 events above $4 \times 10^{19} \mathrm{eV}$ with a total exposure of $\sim 4 \times$ $10^{20} \mathrm{~cm}^{2} \mathrm{ssr}[15$. This translates into an observational super-GZK flux,

$$
I_{>G Z K}^{o b s} \simeq 1.43 \times 10^{-19} \mathrm{~cm}^{-2} \mathrm{~s}^{-1} \mathrm{sr}^{-1}
$$

It can be shown that a fitting spectrum with a power-law index -2.78 [16] reproduces the above flux.

\section{Z-BURST YIELD}

Now we deduce the total Z-burst proton yield within a relevant cosmic volume. Solar and atmospheric data on neutrino oscillations indicate that the oscillation lengths are much shorter than the solar distance. So for cosmic neutrinos their population among the 3 flavors should be equalized. The total UHEC $\nu$ flux is thus simply 3 times that for a single neutrino flavor. We further assume that $\mathrm{UHEC} \nu$ fluxes are the same for neutrinos and antineutrinos. By definition,

$$
\begin{aligned}
I_{p \mid \nu}^{Z} & =3 \xi_{p+n \mid \nu} \int_{0}^{R_{\max }} d r \int_{0}^{\infty} d E F(E, r) \\
& \times \sigma_{\nu \bar{\nu}}\left(E=\frac{s}{2 m_{\nu}}\right) \operatorname{Br}(Z \rightarrow \text { hadrons }) n_{\nu}(r) .
\end{aligned}
$$

Here $F(E, r)$ is the UHEC $\nu$ flux at energy $E$ and distance $r$ from the Earth, $n_{\nu}(r)$ is the number density of the $\mathrm{C} \nu \mathrm{B}, \sigma_{\nu \bar{\nu}}(s)$ the neutrino-antineutrino cross section at $s=2 m_{\nu} E, \operatorname{Br}(Z \rightarrow$ hadrons $)$ the branching ratio, and $\xi_{p+n \mid \nu}$ the multiplicity of nucleons per Z-burst.

For completeness, our integration should include all neutrinos and Z-burst events in the universe. Such a treatment tends to be over-conservative as the protons deduced from Z-bursts outside of our local GZK-sphere may hardly survive. The complete but ultra-conservative treatment is discussed in the appendix. A physically reasonable yet much simplified calculation can be carried out by neglecting the contributions outside of our local GZKsphere. This amounts to replacing the maximum distance $R_{\max }$ in our integration by the radius of our local GZKsphere $\left(R_{G Z K} \sim 50 \mathrm{Mpc}\right)$. As the distance under consideration is much more local, all the $r$-dependence can be ignored:

$$
\begin{aligned}
I_{p \mid \nu}^{Z} & =3 \xi_{p+n \mid \nu} R_{G Z K} n_{\nu} \operatorname{Br}(Z \rightarrow \text { hadrons }) \\
& \times \int_{0}^{\infty} d E F(E) \sigma_{\nu \bar{\nu}}(E),
\end{aligned}
$$

where $n_{\nu}(r) \equiv n_{\nu}=112 \mathrm{~cm}^{-3}$ is the neutrinoantineutrino number density per flavor at present.

The UHEC $\nu$ flux is commonly assumed to follow a power-law energy spectrum

$$
F(E)=F_{0} E^{-\alpha},
$$

with $F_{0}$ being the normalization factor.

Using $E=s E_{\text {res }} / M_{Z}^{2}$, we can write the energy integration as

$$
\begin{aligned}
& \int_{0}^{\infty} d E F(E) \sigma_{\nu \bar{\nu}}(s=2 m E) \\
= & E_{\text {res }} \int_{0}^{\infty} \frac{d s}{M_{Z}^{2}} F\left(s E_{r e s} / M_{Z}^{2}\right) \sigma_{\nu \bar{\nu}}(s) .
\end{aligned}
$$

As the neutrino-antineutrino annihilation cross section is sharply peaked at the Z-resonance, it acts essentially like a $\delta$-function in the integration over the energy of the $\mathrm{UHEC} \nu$. We therefore introduce the energy-averaged cross section [6, 10]

$$
\left\langle\sigma_{\nu \bar{\nu}}\right\rangle \equiv \int \frac{d s}{M_{Z}^{2}} \sigma_{\nu \bar{\nu}}(s)=2 \pi \sqrt{2} G_{F}=40.4 \mathrm{nb},
$$


which is the effective cross section for all neutrinos within the resonance range $\left(E_{\text {res }}\left(1-\Gamma_{Z} / M_{Z}\right), E_{\text {res }}\left(1+\Gamma_{Z} / M_{Z}\right)\right)$ and simplify the integration of Eq. (8) as

$$
\begin{aligned}
& E_{\text {res }} \int_{0}^{\infty} \frac{d s}{M_{Z}^{2}} F\left(s E_{r e s} / M_{Z}^{2}\right) \sigma_{\nu \bar{\nu}}(s) \\
\simeq & F_{0} E_{r e s}^{1-\alpha} \int_{0}^{\infty} \frac{d s}{M_{Z}^{2}} \sigma_{\nu \bar{\nu}}(s) \\
= & F_{0} E_{r e s}^{1-\alpha}\left\langle\sigma_{\nu \bar{\nu}}\right\rangle .
\end{aligned}
$$

Putting everything together we find

$$
\begin{aligned}
I_{p \mid \nu}^{Z} & =R_{G Z K} n_{\nu} \xi_{p+n \mid \nu} F_{0}\left(\frac{M_{Z}^{2}}{2 m_{\nu}}\right)^{1-\alpha} \\
& \times\left\langle\sigma_{\nu \bar{\nu}}\right\rangle \operatorname{Br}(Z \rightarrow \text { hadrons }) .
\end{aligned}
$$

The experimental data [17] gives the branching ratio

$$
\operatorname{Br}(Z \rightarrow \text { hadrons })=(69.89 \pm 0.07) \% .
$$

The final proton multiplicity per Z-burst was calculated by Fodor, Katz and Ringwald(see [18] and references therein) as a function of the proton momentum distribution and by Gelmini, Varieschi and Weiler 12] using the event generator PYTHIA 19]. They obtain $\xi_{p+n \mid \nu} \cong 2.04$ and 1.6 , respectively. We take the former value in this paper.

\section{CASCADE LIMIT AND NEUTRINO MASS BOUND}

We now invoke the cascade limit to constrain the $\mathrm{UHEC} \nu$ flux [20, 21]. This is permissible due to the fact that neutrino productions must always be accompanied by photons and electrons. The cascades are induced while these photons or electrons interact with low energy background radiations such as the $\mathrm{CMB}$ in extra galactic space and the infrared radiation inside the galaxy. The photons so induced would further cascade and eventually pile up in the energy range of $10 \mathrm{MeV}-100 \mathrm{GeV}$ with a spectrum $\propto E^{-2}$, which is consistent with the EGRET observation 22]. The estimated average energy density in this range is $\omega_{\mathrm{EGRET}} \approx 2 \times 10^{-6} \mathrm{eV} / \mathrm{cm}^{3}$. This provides an upper bound on the UHE neutrino flux,

$$
E^{2} F(E)<\frac{c}{4 \pi} \omega_{\text {cas }}<\frac{c}{4 \pi} \omega_{\text {EGRET }}
$$

To be prudent, we do not assume the exact value of $\alpha=2$ for the power-law index, but instead leave $\alpha$ as a free parameter, knowing that its value should be close to 2 . Thus the parameter $F_{0}$ can be substituted with an upper bound as follows:

$$
\begin{array}{ll}
F_{0}<\frac{c}{4 \pi} \omega_{\text {EGRET }} / E_{\min }^{2-\alpha} & , \quad \alpha \geq 2, \\
F_{0}<\frac{c}{4 \pi} \omega_{\text {EGRET }} / E_{\max }^{2-\alpha} \quad, \quad \alpha<2,
\end{array}
$$

where $E_{\max }$ and $E_{\min }$ are the maximum and minimum energies of the UHEC $\nu$ spectrum.
Implementing the cascade limit condition, and inserting all the relevant physical quantities discussed in the previous section, Eq.(2) becomes

$$
\begin{aligned}
& m_{\nu}>28.7\left(\frac{E_{\text {res }}}{E_{\min }}\right)^{\alpha-2} \mathrm{eV}, \quad \alpha \geq 2, \\
& m_{\nu}>28.7\left(\frac{E_{\max }}{E_{\text {res }}}\right)^{2-\alpha} \mathrm{eV}, \quad \alpha<2 .
\end{aligned}
$$

Note that in this expression not all the $m_{\nu}$ dependence were grouped to the LHS, as $E_{\text {res }}$ clearly depends on $m_{\nu}$. Nevertheless this expression has an advantage in that $E_{\text {res }} / E_{\min } \geq 1$ and $E_{\max } / E_{\text {res }} \geq 1$ by definition.

An explicit $m_{\nu}$ lower bound can be obtained by moving all the $m_{\nu}$ dependence to the LHS. We then find

$$
\begin{aligned}
& m_{\nu}>\frac{1}{2}\left(\mathcal{A}^{\frac{1}{\alpha-1}} E_{\min }^{\frac{2-\alpha}{\alpha-1}} M_{Z}^{2}\right), \alpha \geq 2, \\
& m_{\nu}>\frac{1}{2}\left(\mathcal{A}^{\frac{1}{\alpha-1}} E_{\max }^{\frac{2-\alpha}{\alpha-1}} M_{Z}^{2}\right), \alpha<2,
\end{aligned}
$$

where

$$
\begin{aligned}
\mathcal{A}= & I_{>G Z K}^{\text {obs }}\left[\frac{c}{4 \pi} \omega_{\mathrm{EGRET}} n_{\nu} R_{G Z K}\right. \\
& \left.\xi_{p+n \mid \nu}\left\langle\sigma_{\nu \bar{\nu}}\right\rangle \operatorname{Br}(Z \rightarrow \text { hadrons })\right]^{-1} .
\end{aligned}
$$

The mass bound is dependent on the power-law in$\operatorname{dex} \alpha$ and the values of $E_{\max }$ or $E_{\text {min }}$. Our limited knowledge on the $\mathrm{UHEC} \nu$ renders large uncertainty in the determination of $E_{\max }$ and $E_{\text {min }}$. One thing which is certain, however, is that the resonant energy must lie in between $E_{\max }$ and $E_{\min }$ for the Z-burst to happen. Eq.(15) indicates that the minimum value of our bound corresponds to the situation where either $E_{\max }$ or $E_{\min }$ equals $E_{\text {res }}$, or $\alpha=2$. Since we should look for the lowest possible lower bound, we put $E_{\text {res }}=E_{\max }=E_{\text {min }}$ in our estimate and arrive at our neutrino mass lower bound

$$
m_{\nu}>28.7_{-10.6}^{+11.8} \mathrm{eV} \quad\left(R_{\max }=R_{G Z K} \sim 50 \mathrm{Mpc}\right),
$$

where the error comes from fitting the AGASA data [16].

Recent WMAP 23] measurement of the CMB fluctuations has deduced a strong upper limit on neutrino masses, $\Sigma_{i} m_{\nu_{i}}<0.69 \mathrm{eV}$. Since any single neutrino mass $\sim 0.04 \mathrm{eV}$ implies a near mass-degeneracy for all three active neutrinos, one concludes $m_{\nu}<0.23 \mathrm{eV}$. Two analyses [24, 25] which include data from WMAP, 2dF, SDSS, and galaxy cluster surveys have arrived at a bound of $\Sigma_{i} m_{\nu_{i}} \lesssim 0.7 \mathrm{eV}$. Another analysis [26] using CMB and LSS data gives $\Sigma_{i} m_{\nu_{i}} \lesssim 1 \mathrm{eV}$, but finds a stronger bound $\Sigma_{i} m_{\nu_{i}} \lesssim 0.6 \mathrm{eV}$ when priors from supernova data and Hubble Key Project are included. These newer results are close to the original WMAP bound. All these analyses converge to a cosmological upper bound of $m_{\nu} \lesssim$ $0.23 \mathrm{eV}$, which is 2 order of magnitudes smaller than the lowest possible lower bound we have derived. 


\section{IMPLICATION}

Our derivation is based on two assumptions: the saturation of the observed super-GZK UHECR flux by the Z-burst mechanism and the cascade upper limit on the maximum UHE $\nu$ flux.

Since the cascade limit is deduced from the cascades of photons accompanying the neutrino production, it is valid for all sources. Not only astrophysical accelerators (e.g. GRBs, AGNs, SNs, etc.) but also top-down sources, such as topological defects, superheavy $\mathrm{X}$ particles, dark matter, etc., are all contributing to this limit as long as the photons are co-produced along-side with neutrinos. It is generally believed [27, 28] that the cascade limit on UHE $\nu$ flux is quite robust.

Even under the most conservative assumption, $E_{\min }=E_{\max }=E_{\text {res }}$, i.e. that the UHEC $\nu$ spectrum is a delta function, our deduced neutrino mass lower bound is more than 120 times larger than the existing upper bound. We therefore conclude that the Z-burst scenario cannot account for the observed super-GZK UHECR flux. Assuming all the parameters are fixed, our neutrino mass lower bound can be lowered if we allow Z-burst to contribute only partially to the observed UHECR flux. Based on our values we can conclude that the Z-burst contribution to UHECR cannot be more than $\sim 1 \%$ within our local GZK-sphere. The recent ANITA-lite experiment [13] indicates that the Z-burst can at best contribute $\sim 10 \%$ to the UHECR spectrum, which is consistent with our conclusion.

Analogous to the GZK process, Z-burst is one of the few robust cosmic interaction processes based on the standard model of particle physics. With a mean-freepath comparable to the present Hubble radius, it provides much hope to resolve the existing challenge of the bottom-up scenario. Our negative conclusion on its viability as a solution to the super-GZK puzzle seems to force us back to the original dilemma. If the excess superGZK flux is found to be real, the need for a solution remains.

\section{APPENDIX A: A COMPLETE BUT ULTRA-CONSERVATIVE VERSION}

For completeness, the integration over distance must be carried out to include the cosmological evolution of the $\mathrm{C} \nu \mathrm{B}$ number density and the UHEC $\nu$ flux. Since the dependence on the propagation distance $r$ of the $\mathrm{UHEC} \nu$ fluxes and the $\mathrm{C} \nu \mathrm{B}$ number density can be expressed more straight-forwardly in terms of the redshift parameter $z$, we make the following change of variables

$$
d r=-\frac{c H_{0}^{-1}}{(1+z) \sqrt{\Omega_{m}(1+z)^{3}+\Omega_{\Lambda}}} d z
$$

where $\Omega_{m} \approx 0.3$ and $\Omega_{\Lambda} \approx 0.7$ are the present matter and dark energy densities in units of the critical density, respectively.

According to the big bang cosmology, the number density of $\mathrm{C} \nu \mathrm{B}$ is $n_{\nu}(z)=n_{\nu}(1+z)^{3} \mathrm{~cm}^{-3}$, where $n_{\nu}=$ $112 \mathrm{~cm}^{-3}$ is the neutrino-antineutrino number density at present. The UHEC $\nu$ flux is now assumed to follow a power-law energy spectrum with cosmological evolution of the source included [8, 29, 30] and can be parameterized as

$$
F(E, r)=F(E) f(z)=F_{0} E^{-\alpha} f_{0}(1+z)^{\beta},
$$

where $f_{0}$ is the normalization factor for the source evolution determined by the condition

$$
\int_{0}^{z_{\max }} f(z) d z=1
$$

with $z_{\max }$ being the redshift of the most distant source.

A more sophisticated distribution function has been introduced, based on the star formation rate and the GRB site distribution 31]

$$
f(z)=f_{0} \frac{1+a_{1}}{(1+z)^{-a_{2}}+a_{1}(1+z)^{a_{3}}},
$$

where $a_{1}, a_{2}$ and $a_{3}$ are fitting parameters. It can be shown that our neutrino mass bound is insensitive between these two choices of distribution functions and for simplicity we will invoke Eq.(8) in our subsequent discussion. The separation of variables allows us to carry out the UHEC $\nu$ energy and distance integrations independently.

The integration over propagation distance involves several sources of cosmic evolution,

$$
\begin{aligned}
& \int_{0}^{z_{\max }} c H_{0}^{-1} \frac{n_{\nu}(1+z)^{3} f(z) d z}{(1+z) \sqrt{\Omega_{m}(1+z)^{3}+\Omega_{\Lambda}}} \\
\equiv & c H_{0}^{-1} n_{\nu} f\left(z_{\max }, \beta\right),
\end{aligned}
$$

where simple analytic result is not readily attainable. Table đisplays values of $f\left(z_{\max }, \beta\right)$ for selected choices of $z_{\max }$ and $\beta$. It is clear that $f\left(z_{\max }, \beta\right)$ is reasonably insensitive to $z_{\max }$ and $\beta$.

TABLE I: Values of $f\left(z_{\max }, \beta\right)$ for selected $z_{\max }$ and $\beta$.

\begin{tabular}{rr|rrrrrrr}
\hline \hline$\beta$ & -3 & -2 & -1 & 0 & 1 & 2 & 3 \\
\hline \multirow{3}{*}{$z_{\max }$} & 1 & 1.47 & 1.54 & 1.62 & 1.69 & 1.76 & 1.82 & 1.88 \\
\cline { 2 - 9 } & 2 & 1.65 & 1.81 & 2.00 & 2.18 & 2.35 & 2.49 & 2.59 \\
\cline { 2 - 9 } & 3 & 1.73 & 1.98 & 2.27 & 2.56 & 2.81 & 2.99 & 3.12 \\
\hline \hline
\end{tabular}


With the knowledge of $f\left(z_{m z x}, \beta\right)$, the Z-burst yield, Eq.(5), becomes

$$
\begin{aligned}
I_{p \mid \nu}^{Z} & =c H_{0}^{-1} n_{\nu} f\left(z_{\max }, \beta\right) \xi_{p+n \mid \nu} F_{0}\left(\frac{M_{Z}^{2}}{2 m_{\nu}}\right)^{1-\alpha} \\
& \times\left\langle\sigma_{\nu \bar{\nu}}\right\rangle \operatorname{Br}(Z \rightarrow \text { hadrons }) .
\end{aligned}
$$

Correspondingly, the lower bound on neutrino mass is changed to

$$
\begin{aligned}
& m_{\nu}>1.25 f\left(z_{\text {max }}, \beta\right)^{-1}\left(\frac{E_{\text {res }}}{E_{\text {min }}}\right)^{\alpha-2} \mathrm{eV}, \quad \alpha \geq 2, \\
& m_{\nu}>1.25 f\left(z_{\text {max }}, \beta\right)^{-1}\left(\frac{E_{\text {max }}}{E_{\text {res }}}\right)^{2-\alpha} \mathrm{eV}, \quad \alpha<2 .(\mathrm{A} 7)
\end{aligned}
$$

while Eqs.(16) and (17) remain the same form with

$$
\begin{aligned}
\mathcal{A}= & I_{>G Z K}^{o b s}\left[\frac{c}{4 \pi} \omega_{\text {EGRET }} c H_{0}^{-1} n_{\nu}\right. \\
& \left.\xi_{p+n \mid \nu}\left\langle\sigma_{\nu \bar{\nu}}\right\rangle \operatorname{Br}(Z \rightarrow \text { hadrons })\right]^{-1} .
\end{aligned}
$$

The mass bound is now dependent on the numerical values of the evolution factor $f\left(z_{\max }, \beta\right)$ as well (See Table III). Again we look for the lowest possible lower bound by putting $E_{\text {res }}=E_{\max }=E_{\text {min }}$ in our estimate. As we have shown, $f\left(z_{\max }, \beta\right)$ is of the order 1 and is insensitive to $z_{\max }$ and $\beta$. With the choice of $f\left(z_{\max }, \beta\right)=f(3,0)=2.56$, we arrive at our neutrino mass lower bound

$$
m_{\nu}>0.49_{-0.18-0.14}^{+0.20+0.53} \mathrm{eV} \quad\left(R_{\max } \sim c H_{0}^{-1}\right) .
$$

The former error comes again from the AGASA data and the latter from the uncertainty of the evolution factor $f\left(z_{\max }, \beta\right)$.

TABLE II: Values of mass lower bound for selected $z_{\max }$ and $\beta$ with $\alpha=2$ energy spectrum.

\begin{tabular}{crrrrrrrrr}
\hline \hline \multicolumn{110}{c}{$\alpha=2$} \\
\hline \hline$\beta$ & -3 & -3 & -3 & 0 & 0 & 0 & 3 & 3 & 3 \\
$z_{\max }$ & 1 & 2 & 3 & 1 & 2 & 3 & 1 & 2 & 3 \\
$f\left(z_{\max }, \beta\right)$ & 1.47 & 1.65 & 1.73 & 1.69 & 2.18 & 2.56 & 1.88 & 2.59 & 3.12 \\
mass bound & 0.85 & 0.76 & 0.73 & 0.74 & 0.58 & 0.49 & 0.68 & 0.48 & 0.40 \\
\hline \hline
\end{tabular}

\section{ACKNOWLEDGMENTS}

We thank P. Blasi, Je-An Gu and K. Reil for valuable discussions. KCL appreciates guidance and support of W-Y. P. Hwang. This work is supported by the National Science Council (NSC 94-2112-M-002-029; NSC 93-2112-M-002-025) of Taiwan, R.O.C., and in part by US Department of Energy under Contract No. DE-AC03$76 \mathrm{SF} 00515$.
[1] K. Greisen, Phys. Rev. Lett. 16, 748(1966).

[2] G. T. Zatsepin and V. A. Kuzmin, Sov. Phys. JETP Lett. 4, 78(1966)

[3] R. U. Abbasi et al., Phys. Rev. Lett. 92, 151101(2004)

[4] M. Takeda et al., Phys. Rev. Lett. 81, 1163-1166(1998)

[5] M. Takeda et al., Astropart. Phys. 19, 447462(2003) astro-ph 0209422.

[6] T. J. Weiler, Phys. Rev. Lett. 49, 234(1982)

[7] E. Roulet, Phys. Rev. D47, 5247-5252(1993)

[8] S. Yoshida, H. Dai, C. C. H .Jui and P. Sommers, Astrophys. J. 479, 547-559(1997).

[9] D. Fargion, B. Mele and A. Salis, Astrophys. J. 517, 725733(1999).

[10] T. J. Weiler, Astropart. Phys. 11, 303-316(1999).

[11] Z. Fodor, S. D. Katz and A. Ringwald, Phys. Rev. Lett. 88, 171101(2002).

[12] G. Gelmini, G. Varieschi and T. J. Weiler, Phys. Rev. D70, 113005(2004).

[13] P. Gorham, Presentation given at PANIC05: Particles and Nuclei International Conference, Santa Fe, NM, October 24-28, 2005.

[14] K. Shinozaki et al., Astrophys. J. 571, L117-L120(2002).

[15] N. Hayashida et al., Astron. J. 120, 2190(2000) astro-ph/0008102.

[16] M. Nagano and A. A. A. Watson, Rev. Mod. Phys. 72,
689-732(2000).

[17] D. E. Groom et al. [Particle Data Group Colaboration], Eur. Phys. J C15, 1(2000).

[18] Z. Fodor, S. D. Katz and A. Ringwald, JHEP, 06, 046(2002) hep-ph/0203198.

[19] T. Sjostrand, Comput. Phys. Commun. 82, 74-90(1994).

[20] V. S. Berezinsky, S. V. Bulanov, V. A. Dogiel, V. L. Ginzburg and V. S. Ptuskin, Astrophysics of Cosmic Rays, North-Holland, 1990.

[21] V. Berezinsky and A. Smirnov, Ap. Sp. Sci. 32, 461(19)1975.

[22] P. Sreekumar et al. [EGRET collaboration], Astrophys. J. 494, 523-534(1998) astro-ph/9709257.

[23] D. N. Spergel et al. [WMAP collaboration], Astrophys. J. Suppl. 148, 175(2003) astro-ph/0302209.

[24] V. Barger, D. Marfatia and A. Tregre, Phys. Lett. B595, 55-59(2004) hep-ph/0312065.

[25] S. W. Allen, R. W. Schmidt and S. L. Bridle, Mon. Not. Roy. Astron. Soc. 346, 593(2003) astro-ph/0306386).

[26] P. Crotty, J. Lesgourgues and S. Pastor, Phys. Rev. D69, 123007(2004) hep-ph/0402049.

[27] Z. Fodor, S. D. Katz, A. Ringwald and H. Tu, JCAP 0311, 015(2003) hep-ph/0309171.

[28] V. Berezinsky, Presentation at 13th International Symposium on Very High-Energy Cosmic Ray Interactions 
at the NESTOR Institute, Pylos, Greece, 6-12 Sep 2004, astro-ph/0505220.

[29] O. E. Kalashev, V. A. Kuzmin, D. V. Semikoz and S. Gunter, Phys. Rev. D65, 103003(2002) hep-ph/0112351.
[30] S. Yoshida, G. Sigl and S. Lee, Phys. Rev. Lett. 81, 55055508(1998) hep-ph/9808324.

[31] S. D. Wick, C. D. Dermer and A. Atoyan, Astropart. Phys. 21, 125-148(2004) astro-ph/0310667. 